\title{
An IVF pregnancy, a neck lump and shortness of breath
}

\author{
Authors: Kyle F Petrie, ${ }^{A}$ Rebeca MP Carter, ${ }^{B}$ Ash Sadighi, ${ }^{C}$ Andrew MTL Choong, ${ }^{\mathrm{D}}$ Alun H Davies ${ }^{\mathrm{E}}$ and \\ Audrey LA Wong ${ }^{F}$
}

\begin{abstract}
We present the case of a young primigravida, conceived with in vitro fertilisation, referred with unilateral neck swelling and pleuritic chest pain. Our case highlights the potential complexity of the management of the complications of assisted conception techniques. The discussion explores important considerations in the management of such patients.
\end{abstract}

KEYWORDS: In vitro fertilisation, shortness of breath, neck lump

\section{Case presentation}

A 33-year-old gravida 1 para 0 in her seventh week of pregnancy was referred by her general practitioner (GP) to our acute admissions unit with a sudden increase in neck pain, constant in nature and exacerbated by movement, on a background of pain for the preceding three weeks. The neck pain was associated with swelling and pleuritic pain. Furthermore, she noticed a difference in skin pallor between the left and right hands. She described occasional shortness of breath.

She had been reviewed by the assisted conception unit with these symptoms; however, this myriad of symptoms was initially thought to be unrelated to pregnancy. She was advised to seek medical attention from her GP and was commenced on antibiotics for suspected lymphadenitis. However, within 72 hours, she re-attended her GP as there was no symptomatic improvement and at that point, she was referred to hospital.

She had conceived with in vitro fertilisation (IVF) and had previously already suffered from bilateral pleural effusions and ascites in the post-implantation period. She was diagnosed with ovarian hyperstimulation syndrome (OHSS) and commenced on prophylactic dose enoxaparin (40 mg once daily). Regular medications included cyclogest, enoxaparin,

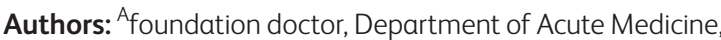

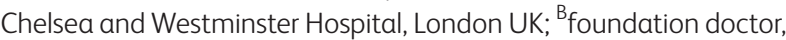
Department of Acute Medicine, Chelsea and Westminster Hospital, London UK; ' registrar, Department of Acute Medicine, Chelsea and Westminster Hospital, London UK; D senior registrar, Imperial Vascular Unit, St Mary's Hospital, London, UK; ${ }^{\text {E}}$ Consultant vascular surgeon, Imperial Vascular Unit, St Mary's and Charing Cross Hospitals, London, UK; F consultant acute medicine physician, Department of Acute Medicine, Chelsea and Westminster Hospital, London, UK folic acid and Pregnacare ${ }^{\circledR}$. She had a family history of pulmonary embolus (PE).

On admission, our patient was haemodynamically stable with oxygen saturations of $99 \%$ on room air. Clinical examination confirmed a swelling of approximately $2 \times 8 \mathrm{~cm}$ in the right posterior triangle of the neck. Blood tests revealed a C-reactive protein of 192; the remainder were normal within the context of pregnancy.

\section{What are the differential diagnoses?}

This patient presented with pleuritic chest pain and worsening neck swelling associated with pain, on a background of OHSS, a condition known to predispose to venous thromboembolism. The sudden onset of pleuritic pain and shortness of breath, in association with OHSS, led to the consideration of a PE; however, clinical observations were stable. Our patient was not hypoxic and not tachycardic. Pulmonary function can be affected during pregnancy secondary to hormonal changes and mechanical obstruction of ventilation by the growing fetus. With this in mind, a physiological response to pregnancy was considered as a further diagnosis.

On examination, there was obvious neck swelling. She had been reviewed by her GP who initiated treatment for lymphadenitis secondary to bacterial illness. While this was a reasonable diagnosis, given her previous diagnosis of OHSS, we felt it was important to exclude jugular vein thrombosis as a cause of worsening neck swelling and pain.

\section{Case progression}

An ultrasound scan (USS) of the neck suggested thrombus within the right internal jugular vein (Fig 1). There was no evidence of subclavian or axillary vein thrombus.

A transvaginal USS (TV-USS) confirmed a viable intra-uterine pregnancy and an area of organised subchorionic haematoma measuring $43 \times 26 \times 27 \mathrm{~mm}$. At this point, we decided not to proceed to computed tomography pulmonary angiogram (CTPA) due to potential risks to the patient and fetus. In addition, given the findings of TV-USS, she would not have been a candidate for thrombolysis. After multidisciplinary team input, including acute care, haematology and obstetrics, the dose of enoxaparin was increased to $60 \mathrm{mg}$ twice daily and she was discharged with follow up. Despite this, she re-presented three days later with acute pleuritic chest pain and worsening dyspnoea. An echocardiogram was normal with no evidence of right heart strain. 


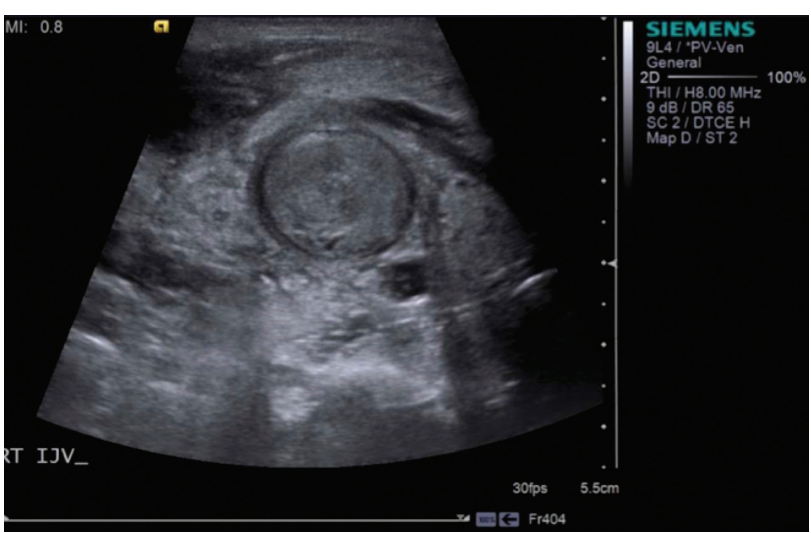

Fig 1. Ultrasound scan of the neck suggesting right internal jugular vein thrombus.

After extensive multidisciplinary discussion, particularly in view of worsening chest pain, we proceeded to CTPA. This demonstrated filling defects within the left interlobar and right subsegmental pulmonary arteries confirming bilateral PEs (Fig 2).

The patient transferred to our tertiary vascular surgery centre for further management. Lower limb duplex USS gave no evidence of further emboli and a superior vena caval filter was sited with a view for removal post-delivery (Fig 3 ). She was discharged with an increased dose of enoxaparin (60 mg three times daily) titrated against anti-Xa levels and outpatient follow up.

She has had no further admissions and delivered at full term with no complications.

\section{Discussion}

OHSS occurs as a result of ovulation induction secondary to gonadotrophin administration during assisted conception techniques. ${ }^{1,2}$ The incidence of OHSS ranges from 0.6 to $14 \%$ of all stimulation cycles. ${ }^{3}$ Risk factors include polycystic ovaries, low body mass index, women under the age of 30, use of human chorionic gonadotropin to stimulate ovulation and previous diagnoses of OHSS. ${ }^{4,5}$

The prevalence of infertility has increased due to delayed age of childbearing, greater incidence of sexually transmitted infections and obesity. Consequently, incidence of OHSS is on the rise due to increased use of assisted conception. ${ }^{6,7}$ This is associated with an increase in presentation to GP surgeries

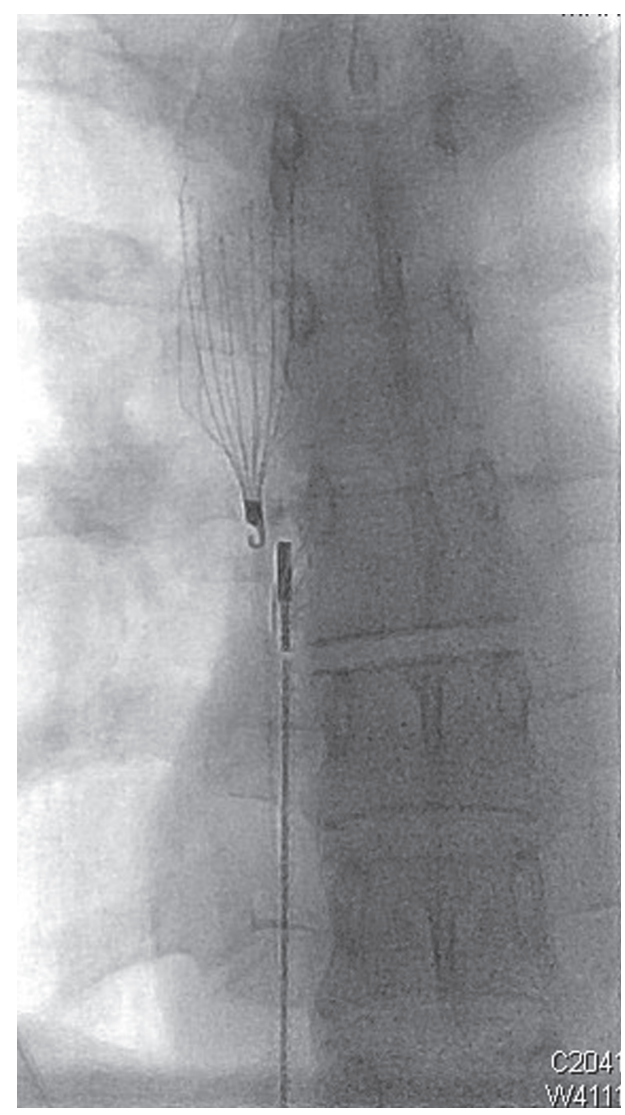

Fig 3. Insertion of a superior vena caval filter.

and acute care services. ${ }^{8,9}$ Despite this, there is a lack of evidence-based medicine and no current consensus on clinical management. ${ }^{5}$

OHSS is a syndrome of clinical features including venous thromboembolism, pleural effusion, ascites, renal failure and electrolyte disturbance $e^{4,8-11}$ and is associated with miscarriage and premature delivery. ${ }^{12}$ It is classified based on the severity of symptoms. $^{4,11,13}$

Venous thromboembolism is the most serious and commonly recognised complication of severe OHSS affecting between $7-10 \%$ of cases. An unexplained predominance for upper limb thrombus has been documented. ${ }^{14}$ OHSS has been documented to confer a 100 -fold increase in thrombosis risk. ${ }^{8}$

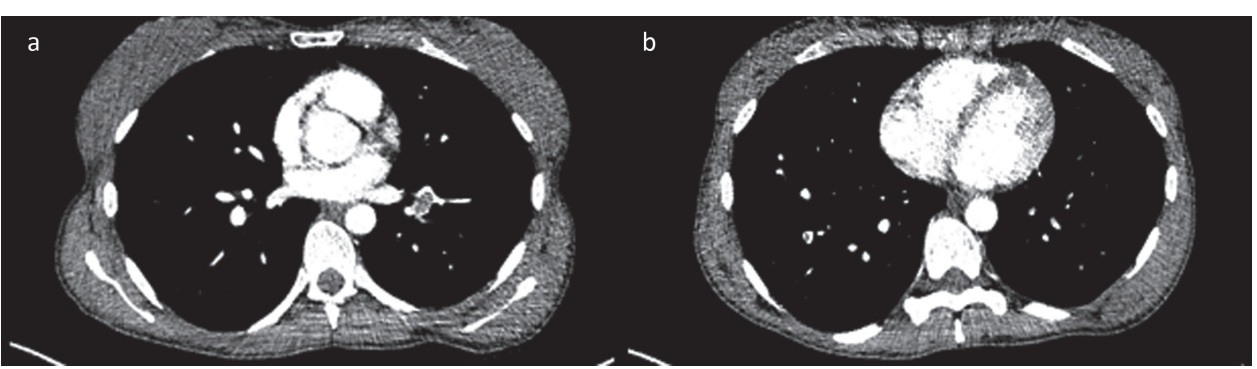

Fig 2. Bilateral pulmonary emboli on computed tomography pulmonary angiogram. 
Increased risk of venous thrombosis is thought to persist into the first trimester. In view of this, the Royal College of Obstetricians and Gynaecologists recommend that all women with OHSS are commenced on prophylactic doses of lowmolecular weight heparin (LMWH) at least until the end of the first trimester. ${ }^{4} \mathrm{LMWH}$ is safe to use in pregnancy as it does not cross the placenta. ${ }^{15}$

If $\mathrm{PE}$ is suspected, guidance recommends that chest X-ray, electrocardiogram and compression USS of the lower limbs are performed. If these investigations are inconclusive, use of ventilation/perfusion (V/Q) scanning or CTPA is indicated. ${ }^{4}$

Pleural effusion and ascites in OHSS are managed effectively with thoracentesis and paracentesis.

OHSS is associated with significantly higher rates of miscarriage, premature delivery and low birth weight in comparison to the general IVF population. In addition, severe OHSS correlates with increased rates of pregnancyinduced hypertension, gestational diabetes and placental abruption. ${ }^{12}$ Despite this, the importance of early recognition and management to reduce maternal morbidity and improve obstetric outcome is clearly evident.

OHSS is a relatively common complication of assisted conception; however, awareness is poor among primary and acute care services. As highlighted here, early recognition and multidisciplinary management confers optimum maternal and obstetric outcome.

\section{Key learning points}

$>$ OHSS is an important complication of IVF of increasing incidence.

$>$ It is a syndrome of clinical features including VTE, ascites and pleural effusion.

> There is a considerable lack of guidance of optimum management, and consequently, poor awareness of the condition.

$>$ Early multidisciplinary team input is vital in the management of such cases.

\section{References}

1 Zivi E, Simon A, Laufer N. Ovarian hyperstimulation syndrome: definition, incidence, and classification. Semin Reprod Med 2010;28:441-7.
2 Calabro RS, Gervasi G, Leo A et al. Neurovascular complications of ovarian hyperstimulation syndrome (OHSS): from pathophysiology to recent treatment options. Recent Pat Endocr Metab Immune Drug Discov 2014;8:109-16.

3 Delvigne A, Rozenberg S. Epidemiology and prevention of ovarian hyperstimulation syndrome (OHSS): a review. Hum Reprod Update 2002;8:559-77.

4 Royal College of Obstetricians and Gynaecologist. The management of ovarian hyperstimulation syndrome. London: RCOG, 2006.

5 Joint Society of Obstetricians and Gynaecologists of CanadaCanadian Fertility Andrology Society Clinical Practice Guidelines Committee; Reproductive Endocrinology and Infertility Committee of the SOGC; Executive and Council of the Society of Obstetricians; Gynaecologists of Canada; Board of the Canadian Fertility and Andrology Society, Shmorgun D, Claman P. The diagnosis and management of ovarian hyperstimulation syndrome. $J$ Obstet Gynaecol Can 2011;33:1156-62.

6 Vloeberghs V, Peeraer K, Pexsters A, D’Hooghe T. Ovarian hyperstimulation syndrome and complications of ART. Best Pract Res Clin Obstet Gynaecol 2009;23:691-709.

7 Avraham S, Seidman D. The multiple birth epidemic: revisited. J Obstet Gynaecol India 2012;62:386-90.

8 Nelson SM. Venous thrombosis during assisted reproduction: novel risk reduction strategies. Thromb Res 2013;131:S1-S3.

9 Chan WS, Ginsberg JS. A review of upper extremity deep vein thrombosis in pregnancy: unmasking the 'ART' behind the clot. $J$ Thromb Haemost 2006;4:1673-7.

10 Madill JJ, Mullen NB, Harrison BP. Ovarian hyperstimulation syndrome: a potentially fatal complication of early pregnancy. J Emerg Med 2008;35:283-6.

11 Kumar P, Sait SF, Sharma A, Kumar M. Ovarian hyperstimulation syndrome. J Hum Reprod Sci 2011;4:70-5.

12 Abramov Y, Elchalal U, Schenker JG. Obstetric outcome of in vitro fertilized pregnancies complicated by severe ovarian hyperstimulation syndrome: a multicenter study. Fertil Steril 1998;70:1070-6.

13 Alper MM, Smith LP, Sills ES. Ovarian hyperstimulation syndrome: current views on pathophysiology, risk factors, prevention, and management. J Exp Clin Assist Reprod 2009;6:3.

14 Kasum M, Danolic D, Oreskovic S et al. Thrombosis following ovarian hyperstimulation syndrome. Gynecol Endocrinol 2014;30:764-8.

15 Nelson SM, Greer IA. Artificial reproductive technology and the risk of venous thromboembolic disease. J Thromb Haemost 2006;4:1661-3.

Address for correspondence: Dr K Petrie, Department of Acute Medicine, Chelsea and Westminster Hospital, 349 Fulham Road, London SW10 9NH, UK.

Email: kyle.petrie@doctors.org.uk 